\title{
GAS PHASE CHEMISTRY IN COMETS
}

M. Oppenheimer

Present theories for the formation of molecular species observed in comets predict the sublimation of parent molecules such as $\mathrm{H}_{2} \mathrm{O}, \mathrm{CH}_{4}, \mathrm{CO}_{2}$, and $\mathrm{NH}_{3}$ from the surface of the nucleus and their subsequent photodissociation and ionization to form the observed species (Delsemme 1973). It can be shown (Oppenheimer 1975) that gas phase chemical reactions occur between these fragments which have characteristic timescales which are short compared to the timescale for significant variation in the solar flux incident on the comet. Hence, a steady-state approximation may be used for determining the densities of many species. It can also be shown (Oppenheimer 1975) that the rate of formation of many species is faster by gas phase reactions than by photoprocess. For instance, the formation of $\mathrm{OH}^{+}$from $\mathrm{H}_{2} \mathrm{O}^{+}$by the reaction

$$
\mathrm{H}_{2} \mathrm{O}^{+}+\mathrm{O} \rightarrow \mathrm{OH}^{+}+\mathrm{OH}
$$

with an estimated rate coefficient of $1 \times 10^{-9} \mathrm{~cm}^{3} \mathrm{~s}^{-1}$ proceeds more rapidly than by the process

$$
\mathrm{H}_{2} \mathrm{O}^{+}+\mathrm{h \nu} \rightarrow \mathrm{OH}^{+}+\mathrm{H}
$$

if the density of atomic oxygen exceeds $10^{4} \mathrm{~cm}^{-3}$ at heliocentric distance $r=1 \mathrm{AU}$. Hence, gas phase reactions 
rapidly reshuffle parent molecules and their fragments in the coma.

The reaction sequence leading to the formation of $\mathrm{H}_{2} \mathrm{O}$ illustrates the significance of gas phase reactions in determining the nuclear structure. Molecular hydrogen will form if the nucleus is composed of almost any hydrogenbearing compound. If oxygen evolves from the nucleus in any form and is subsequently ionized, the reaction sequence

$$
\begin{aligned}
& \mathrm{O}^{+}+\mathrm{H}_{2} \rightarrow \mathrm{OH}^{+}+\mathrm{H} \\
& \mathrm{OH}^{+}+\mathrm{H}_{2} \rightarrow \mathrm{H}_{2} \mathrm{O}^{+}+\mathrm{H} \\
& \mathrm{H}_{2} \mathrm{O}^{+}+\mathrm{H}_{2} \rightarrow \mathrm{H}_{3} \mathrm{O}^{+}+\mathrm{H} \\
& \mathrm{H}_{3} \mathrm{O}^{+}+\mathrm{e} \rightarrow \mathrm{OH}+\mathrm{H}_{2} \\
& \rightarrow \mathrm{H}_{2} \mathrm{O}+\mathrm{H}
\end{aligned}
$$

leads to the formation of the observed cometary species $\mathrm{OH}$, $\mathrm{OH}^{+}$, and $\mathrm{H}_{2} \mathrm{O}^{+}$(Delsemme 1973; Wehinger et al. 1974) and $\mathrm{H}_{2} \mathrm{O}$ (Jaçkson et al. 1974). Therefore, an observation of $\mathrm{H}_{2} \mathrm{O}^{+}$ and $\mathrm{H}_{2} \mathrm{O}$ is not sufficient to indicate the composition of the nucleus. If $n(i)$ is the density of constituent i and $\gamma$ is the branching ratio between $\mathrm{OH}$ and $\mathrm{H}_{2} \mathrm{O}$ formation from $\mathrm{H}_{3} \mathrm{O}^{+}$, we find at 0.6 au that

$$
\frac{\mathrm{n}\left(\mathrm{H}_{2} \mathrm{O}^{+}\right)}{\mathrm{n}\left(\mathrm{H}_{2} \mathrm{O}\right)}=3 \times 10^{4} / \mathrm{n}\left(\mathrm{H}_{2}\right) \gamma
$$


if gas phase reactions determine the molecular densities, and

$$
\frac{\mathrm{n}\left(\mathrm{H}_{2} \mathrm{O}^{+}\right)}{\mathrm{n}\left(\mathrm{H}_{2} \mathrm{O}\right)}=\left(\mathrm{n}(\mathrm{e})+10^{-3} \mathrm{n}\left(\mathrm{H}_{2}\right)\right)^{-1}
$$

if $\mathrm{H}_{2} \mathrm{O}$ is sublimated from the nucleus and subsequently ionized.

Gas phase reaction sequences can be formulated which lead to substantial abundances of all molecular species observed in comets if all the necessary atoms are present in any molecular form in the nucleus (Oppenheimer 1975). However, we predict that little or no $\mathrm{CH}_{4}$ and $\mathrm{NH}_{3}$ form in the gas phase compared to radical fragments of these molecules. Many of the reactions in our scheme are of the ionmolecule type and proceed at the gas kinetic rate. Others are of the neutral-neutral type and depend strongly on the particle temperatures. The rates of the latter reactions such as

$$
\mathrm{H}_{2}+\mathrm{O} \rightarrow \mathrm{OH}+\mathrm{H}
$$

may be greatly enhanced if one of the reactants has excess kinetic or internal energy resulting from formation by photodissociation of a molecular parent. The energy of molecular fragments may also be enhanced if they are produced in strongly exothermic reactions. 
Our conclusion is that the effects of gas phase chemical reactions must be considered in interpreting cometary spectra with regard to implications for the structure of the nucleus.

References

A.H. Delsemme 1973 Space Science Reviews 15, 89.

W.M. Jackson, T. Clark, B. Bonn 1974 I.A.U. Circ. No. 2674.

M. Oppenheimer 1975 Ap. J. 196, 251.

P.A. Wehinger, S. Wycoff, G.H. Herbig, G. Herzberg, and H. Lew $1974 \mathrm{Ap}$. J. (Letters) to be published. 


\section{DISCUSSION}

A. H. Delsemme: At the 1965 Comet Colloquium in Liege, I showed that water vaporization leads to a large collisional zone within the inner coma that I called the "chemical" coma. As an illustration of what could happen in the chemical coma, I computed a simple-minded model using thermal equilibrium, and the absorption of the solar light by water as a source of heat. A water, methane plus ammonia coma model leads then to a surprisingly large amount of those parent molecules that are needed to explain the spectra. But the model succeeded in getting rid of the hydrogen excess, by using an unrealistically high temperature. It is unrealistic because the coma is not optically thick and radiates backwards to space by rotational transitions. I should have been wiser: this high temperature could have been avoided by using $\mathrm{HCN}$ and $\mathrm{CO}_{2}$ instead of $\mathrm{CH}_{4}$ and $\mathrm{NH}_{3}$. However, I have never considered that thermal equilibrium was the final answer, and I am glad to see that people are now willing to consider this gas phase chemistry as a proper approach. The individual reactions must now be considered each individually, and the problem becomes formidable, but it is worthwhile trying. I want to encourage Dr. Oppenheimer in his difficult endeavor, by suggesting that he should follow the same way as mine, that is, getting rid of the unobserved methane and ammonia in his future models, in favor of the observed $\mathrm{H}_{2} \mathrm{O}, \mathrm{HCN}$ and $\mathrm{CH}_{3} \mathrm{CN}$, and possibly $\mathrm{CO}$ and $\mathrm{CO}_{2}$ (from the observed $\mathrm{CO}^{+}$and $\mathrm{CO}_{2}{ }^{+}$).

E. Gerard: You say that ion-molecule reactions play an important role in comets (as they seem also in the interstellar medium), so can you give a figure of the electron density needed near the nucleus?

Do you think that such high ion densities can be found very close to the nucleus?

M. Oppenheimer: If you have sublimation rates of neutrals of $10^{6}$ to $10^{7}$, then you do derive ion densities which are the same as the electron density, of $10^{3}$ or $10^{4}$, which is in good agreement with some observations that were made years ago, there was an article by Arpigny in 1965 that mentioned that kind of number.

I haven't seen much since then. But that would be roughly the electron density.

E. Gerard: But you think it is no problem to create these ions as close to the nucleus as you are saying? They can be created very fast?

M. Oppenheimer: There is a peak in the density-this was shown by Jackson, he tells me, some nine years ago. And it is possible to create a very high abundance, not a relative abundance, but an absolute abundance of ions-at few hundred kilometers by photoionization. But they are being removed quickly, of course. 


\section{DISCUSSION (Continued)}

This is without considering chemistry. This is just considering the ionization rates. When you consider the chemistry, it will bring that peak down, because the ions moved.

But you can definitely create a substantial abundance of ions at small distances from the nucleus.

G. H. Herbig: I gather from your abstract that you have a predicted model of the coma, with all your predictions so that we can compare with observations.

M. Oppenheimer: No. There is an article I wrote which will be in Ap. J. in February, which has a model of the coma, based on a methane nucleus, but where all the other atoms are present in some unknown form.

But I don't want that taken seriously in terms that I really believe methane is in the nucleus. I did it to show what would happen if methane were in the nucleus. You could get everything else anyway.

So, that, I am not ready to predict what the nucleus is really made out of, because it is too early. You need more production rates. In a few years, when those production rates a re available when we have a whole table of them, we can make ratios. Then I think from the gas phase chemistry you can say something more intelligent.

The only things you could say now, for instance, are what other people have said-that because, for instance, $\mathrm{CN}, \mathrm{C}_{3}, \mathrm{C}_{2}$ appear early and in great abundance, that that suggests that some hydrocarbon is in there.

And I am ready to go along with that on the basis of what I can say from the chemistry. But more than that is hard to say.

G. H. Herbig: Do you expect appreciable numbers of negative ions, $\mathrm{C}_{2}-$, $\mathrm{OH}-$. Are you concerned about this?

M. Oppenheimer: No, because in the solar radiation field, the photo detachment rates are very, very fast. I would expect that negative ions form but they destroy very quickly. First of all, they form slowly, much slower than positive ions, and they are destroyed much more easily because of detachment in the solar field.

So that I would expect negative ions not to play a big role.

B. Donn: Your analysis suggests a number of new species for which we need the spectrum so that we can try to identify and look for them.

So, in case Dr. Herzberg is running out of work to do-. 


\section{DISCUSSION (Continued)}

\section{(Laughter.)}

W. I. Axford: One cannot talk in terms of a "scale length" for coma ions such as $\mathrm{CO}^{+}$simply because ions (and electrons) do not expand in a more-or-less simple way as do neutrals. In effect the motion of charged particles must be largely determined by the magnetic field surrounding the comet, and by the dynamical effects of the solar wind. Accordingly one can expect the distributions of coma ions to be quite complicated, and certainly not similar to that of neutrals.

The maxima density of ions is determined approximately from the fact that the maximum coma plasma pressure must be comparable to the solar wind ram pressure. This requires maximum ion density of $10^{4}-10^{5} \mathrm{~cm}^{-3}$ depending on their temperatures.

M. Oppenheimer: There is no doubt that the ion distribution is not correct, because it is assumed the ion velocity and the neutral velocities are the same, which won't be true, especially as you get more and more towards the edge.

But, you had to start somewhere. And what it shows is that the ion scale lengths, for instance, may not be determined at all. And even the neutral scale lengths are affected. The $\mathrm{OH}$ scale length that was derived from the observations that were put on earlier was about $10^{5}$ kilometers.

The OH scale length due to chemical processes is comparable to and actually shorter than that. There is a big warning here that you can't assume that the scale lengths that are observed are due to photo processes. Because the chemical processes change the scale lengths all a round.

C. Cosmovici: Does the interaction between neutrals and dust particles near the nucleus become important for the formation of new molecules like in the interstellar medium?

M. Oppenheimer: The interaction between dust particles and neutrals in a strong ionizing field is much slower than the interaction between ions and neutrals.

And the dust particles may effect the distribution but at these temperatures, you don't even expect the neutrals to stick to the dust particles very well. If things are flying off, then they are not coming back and sticking.

So, in terms of what I know, I don't expect them to be as important.

D. A. Mendis: Regarding the conversion of scale lengths to lifetimes even in the case of neutrals one has to be careful especially when they are small (i. e. , $\lesssim 5.10^{4} \mathrm{~km}$ ) because of the effect of collisions. For instance if the expansion is 


\section{DISCUSSION (Continued)}

sonic it means that by the time an emitter goes out a distance $D$, it has also random walked a distance $D$ so already a factor of two is involved.

I would also like to state that with all this talk about the importance of collisions and the gas phase chemistry it is becoming clear that we have to use a complete multiconstituent hydrodynamic model using a proper energy equation before we can get to a proper interpretation of the observations. There is no use trying to fit a Haser model to the exosphere while ignoring the nuclear region-surely what happens in the exosphere is directly related to what happens in the collision dominated region, and a proper hydrodynamic model can be applied to the entire region-including the collisionless region-if properly interpreted.

Also the effect of the attenuation of the incoming exciting radiation in the coma has to be taken into account in a consistent way.

M. Oppenheimer: In line with that point, there is an important one which I want to show.

Reactions like this which make destroy parent molecules themselves very rapidly, are generally ignored, because they have rate constants which aren't high until you get to a few thousand degrees, at which time they do get to be gas kinetic.

The trouble with that is that oxygen coming off is photo dissociated, and may have several volts of energy associated with it. And if $\mathrm{CH}_{4}$, for instance, were a major constituent of the coma, in the first collision oxygen has, instead of being thermalized, it reacts. And "whamo," you have a reaction immediately, which removes one of these what might be a parent molecule.

So even the neutral reactions could be extremely important if we find the photo dissociation products are really hot.

B. Donn: I would like here to make a contribution of my own on this subject, which I mentioned earlier, concerning chemistry in these astronomical sources, and that is: one has to be careful in using laboratory data and applying it here, because the laboratory data is taken under fairly high densities where collisions are frequent.

There, we have a Boltzmann distribution of the vibrational and rotational energy of the participating species in addition to a Maxwellian velocity distribution, which is not true at the densities in the comet. Densities of $10^{6}$ to $10^{12}$ will lead to radiation of the vibrational energy and much of the time rotational energy and you will end up with cold molecules in their ground vibrational states and frequently ground rotational states also. 


\section{DISCUSSION (Continued)}

Almost all of these neutral processes, and some of the ionic processes are very dependent on having vibrationally excited molecules. The reactions take place in excited states, and therefore, one needs to know what the detailed rate constants are, not for a Boltzmann distribution of energies, but for reactions from each of the energy levels, to determine how much the contribution will be made there. Dr. Oppenheimer just gave an example of nonequilibrium velocities and some consequences.

Another point is that in discussing neutral chemical processes, at temperatures of 300 degrees Kelvin or even 500 degrees as Delsemme proposed earlier for most of these processes, the rates are extremely slow. In the time scale of the solar system, you would generally not reach equilibrium.

M. Oppenheimer: Thermal equilibrium has nothing to do with this.

D. J. Malaise: When observing the variations of the photometric profiles with heliocentric distance with high space resolution, several radicals show a behavior which is at complete variance with any kind of photodissociation production.

These radicals show a flatter profile when distance to the sun decreases. In 1966 (Malaise, XIIIth Colloquium of Liege, 199; 1966) I interpreted this as an indication that the production of radicals depended on collisions. At that time, however, the total density of gas at $10^{4} \mathrm{~km}$ from the center was thought to be in the range of $10^{4} \mathrm{~cm}^{-3}$. To solve this contradiction, I looked carefully to find collisional effect in the rotational structure of $\mathrm{CN}$ and to my surprise I found total densities in the range of $10^{8} \mathrm{~cm}^{-3}$ for an average comet. The difficulty is that for small comets (Encke) the total density is $10^{5} \mathrm{~cm}^{-3}$ or smaller. But the small comets produce the same radicals as the large ones.

M. Dubin: Two comments: first in regard to Malaise.

It has been shown that there are two classes of dust particles-small particles and larger particles which sublimate. The distribution of the larger particles may affect the radial dependence on the distributions of the molecules and radicals.

Secondly, Oliveso of Penn. State has been studying the possible role of small dust particles in the Earth's D and E region in relation to the ion chemistry. He has found that at the very small dust concentrations in the Earth's atmosphere the dust reaction is competitive with the ion reaction rates-as the cross section of surface ion recombination is nearly 100 per cent efficient. For comets, with a tremendously larger dust to gas ratio than the Earth's D region this type of reaction must be considered. 


\section{DISCUSSION (Continued)}

M. Oppenheimer: I am very skeptical about those kinds of assumptions, though, because I think that especially in the thermosphere, where the temperatures are a thousand degrees for the neutrals and greater for the ions sometimes, that they don't stick to grains. It just seems unlikely, especially in the light of the kind analysis in the interstellar medium that Salpeter did, that these effects really lead to reactions anywhere near as a efficient as the gas phase.

And I haven't seen that paper, so maybe I should shutup about it.

M. Dubin: D region-

M. Oppenheimer: Oh, the $\mathrm{D}$ region. There it may be a different story.

L. Biermann: I would like to congratulate Dr. Oppenheimer on his very fine work which in many details goes beyond our own work mentioned earlier (Biermann and G. Diercksan, 1974, loc. cit.). His general conclusions are essentially identical with those reached there. As to details, the rate of dissociative recombination of $\mathrm{CO}^{+}$is known from laboratory work since 1970 and was applied to cometary chemistry already then (cf. L. Biermann, Report of IAU Commission 15, 1970). A first value for the electron density in the coma was derived in 1964 in Dr. Trefftz's and my paper quoted by Dr. Delsemme this morning, in which the effect of the absorption of the solar ultraviolet was at least crudely allowed for.

B. Donn: After the reviews by Delsemme and Malaise, the report just presented by Oppenheimer and the discussion by several participants following these three papers and others, it is my feeling that the study of the coma is entering a new level of sophistication. The simple Haser model served for two decades as a valuable scheme for analyzing coma photometry. It now appears that both theory and observation indicate that we must be careful in deducing cometary parameters, e.g., lifetimes and sources from the Haser model. Several refinements need to be added; velocity distribution of fragments, effects of collision, chemical reactions, time variations, more complete hydrodynamic analysis including ions and other processes still to be appreciated. How to do all this is not clear. 